\title{
Interfacing Apple and IBM computers for the analysis of clinical biofeedback
}

\author{
GEORGE ALAN BROWN and FRANCISCO I. PEREZ \\ Baylor College of Medicine, Houston, Texas
}

\begin{abstract}
This paper demonstrates a method of transferring research data from a remote clinic to a large university mainframe for data manipulation and statistical analysis. Data collected by an Apple //e computer were transferred to an IBM 3031 mainframe by sending data files to an IBM PC by telephone modem or by direct hardwire connection to the PC. The IMB PC performed dataformatting routines and then uploaded the files to the mainframe for storage. Advantages and disadvantages of sending data over telephone lines via a modem are discussed.
\end{abstract}

There recently has been some controversy over the efficacy of biofeedback as a treatment for various medical and psychological disorders (Miller, 1982). The literature abounds with single case studies demonstrating the success of the treatment, whereas controlled laboratory studies of groups often have obtained mixed results (Kewman \& Roberts, 1983). Steiner and Dince $(1981,1983)$ have pointed out the problems one faces in trying to equate biofeedback in the laboratory and biofeedback in the clinic. They have reported that many studies in the literature that have not found biofeedback to be effective or more effective than relaxation alone have been poorly controlled. Many times, an effect may be clinically significant but fails to reach statistical significance, especially in group designs. Part of this problem may be due to the fact of the inherent variability of the physiological response that is unique to the individual (Carroll \& PhysDavies, 1979). Although intrasubject patterns seem to be consistent, intersubject correlations may indeed be quite small (Laveren \& Lubin, 1972). This is further supported by the work of Bremner, Yost, and McKenzie (1982), who found consistency in EEG data across days using a single-subject approach. When the data were analyzed as a group design, differences in treatments were washed out. A careful method of applying single-subject statistics has been demonstrated by researchers at Trinity University in San Antonio, Texas (Bremner et al., 1982; Brown, 1982). The single-subject design's power is in its sensitivity in detecting differences when differences in treatments actually exist (Brown, 1982).

After demonstration of this technique in the laboratory, we decided to use these methods to investigate the efficacy of biofeedback in a clinical setting. Although the biofeed-

We gratefully acknowledge the assistance of Cliff Zintgraph, Steve Samples, and Charles Fox for their contributions in the completion of this sudy. George Alan Brown is with the Department of Physical Medicine, and Francisco I. Perez is with the Department of Physical Medicine and the Department of Neurology, Baylor College of Medicine, Houston, TX 77030. Reprint requests should be addressed to: Francisco I. Perez, 6560 Fannin, Suite 1224, Houston, TX 77030. back data-acquisition system was driven by an Apple //e computer, it soon became apparent that the complicated statistical analyses needed for this research were beyond the capability of our Apple computer. It was at this time that a collaborative effort was arranged with Trinity University, approximately 200 miles from our clinic in Houston. It is the purpose of this paper to demonstrate the manner in which data from a remote site may be transferred to a mainframe for statistical analysis.

\section{METHOD}

\section{Subject}

The subject was a 21-year-old female who was referred to the clinic for biofeedback as a treatment in the management of severe headaches. The subject's electromyographic (EMG) activity was recorded and fed back via a loudspeaker. Peripheral skin temperature was also monitored. Descriptions of sensor placements were detailed elsewhere (Brown \& Perez, 1985).

\section{Apparatus}

The EMG activity was recorded by an Autogen 1700 with a bandwidth of 100-200 Hz. An analog tone that fluctuated with fluctuations in EMG served as the feedback. The output of the EMG signal was sent to a Cyborg Biolab external interface module before being fed into a 12 bit A/D converter board contained in an Apple //e computer slot. The Apple //e controlled the data acquisition and stored the digitized trial data to a 20-MB Corvus hard disk. The patient's EMG activity was monitored by a $\mathrm{CRT}$, and the ongoing digitized activity was displayed on a video monitor. (A diagram is illustrated in Figure 1.)

\section{Procedure}

The subject completed the following conditions: visual imagery training and EMG biofeedback training. The patient's baseline was measured before each task and immediately after the second treatment condition. Twelve seconds of data were collected under each condition in 


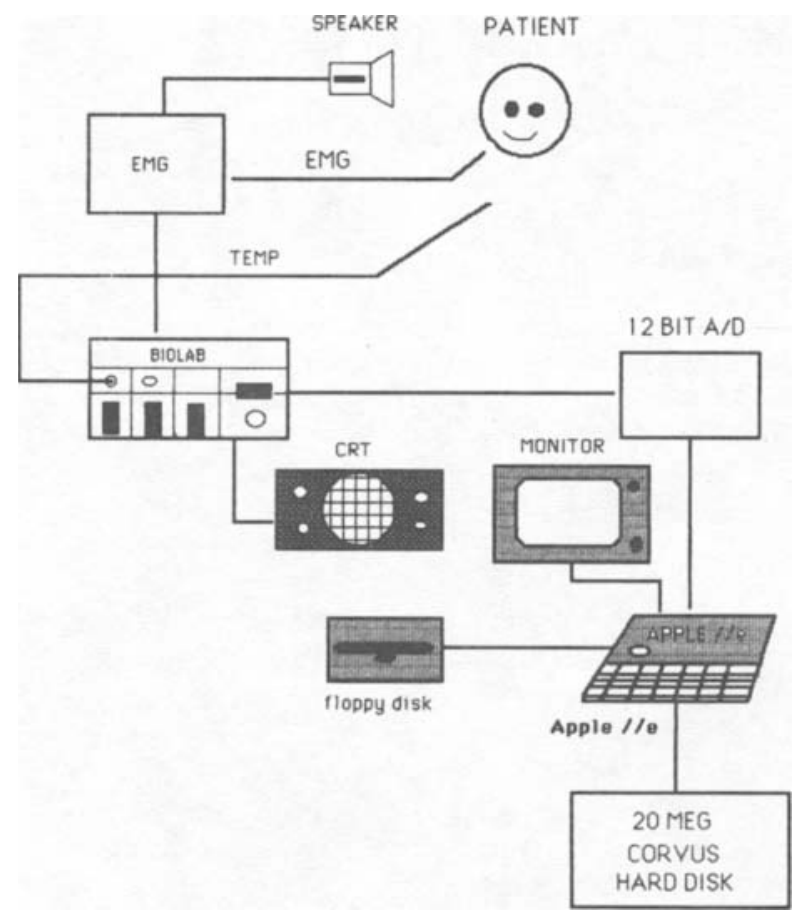

Figure 1. Computer-controlled experimental recording diagram.

two 6-sec blocks. Both physiological signals were sampled at a rate of 256 times a second, for a total of 3,072 samples per condition. Due to limitations of the Apple's memory, the data were sampled in two 6-sec blocks. The data were immediately stored as a text file on a Corvus hard disk for the remainder of the session. After the end of the session, the data were downloaded to a 51/4-in. floppy diskette for archival backup.

\section{Data Transmission 1}

Transmission of data to the mainframe computer was accomplished over telephone line by a 300 -baud Hayes Micromodem II running VisiTerm communications program (Personal Software). An IBM PC running EasyLink software intercepted the text files. The IBM PC was connected to an IBM 3031 mainframe computer, which provided storage and statistical manipulation of the data. A diagram of the communication network is illustrated in Figure 2.

\section{Results}

It was demonstrated that the network of Apple /e, IBM PC, and IBM 3031 could reliably handle the clinic data and transport them to the mainframe. However, at 300 baud, the number of data points to be transferred (120,000 for the eight sessions) would have taken approximately $64 \mathrm{~h}$. This quickly becomes a financial nightmare, especially if more than one subject is used. We decided to implement a faster link.

\section{Data Transmission 2}

Each day's data were downloaded to a floppy disk. The Apple DOS 3.3 diskette was inserted into an Apple computer. Communications software (VisiTerm) transferred the biofeedback data a file at a time $(3,072$ data points) through a serial port hardwired to the serial port of an IBM PC. The data transfer took place at a rate of 9600 baud. The IBM received the data and stored it on an IBM DOS formatted diskette.

At this point, a BASIC program on the IBM PC was used to remove header information (name, condition, instrument settings, and date) from each file and transfer the data directly to an IBM 3031 mainframe running the CMS environment. Once the data files were stored on the cylinder, a program was used to reformat the data to be used in the SAS statistical program. The direct 9600-baud network is illustrated in Figure 3.

\section{Results}

Transmission of data was greatly enhanced by transmitting the data directly from the Apple to the IBM PC via the serial ports. The transfer time was reduced considerably by being able to increase speed to 9600 baud.

\section{DISCUSSION}

The introduction of microcomputers in clinical settings provides an economical method for precise, systematic data collection and storage for most treatment applications. Many data-acquisition systems for biofeedback use an Apple II or Apple //e computer to control data recording, perform intermediate calculations on the data, and provide for feedback to the patient in either the visual or au-

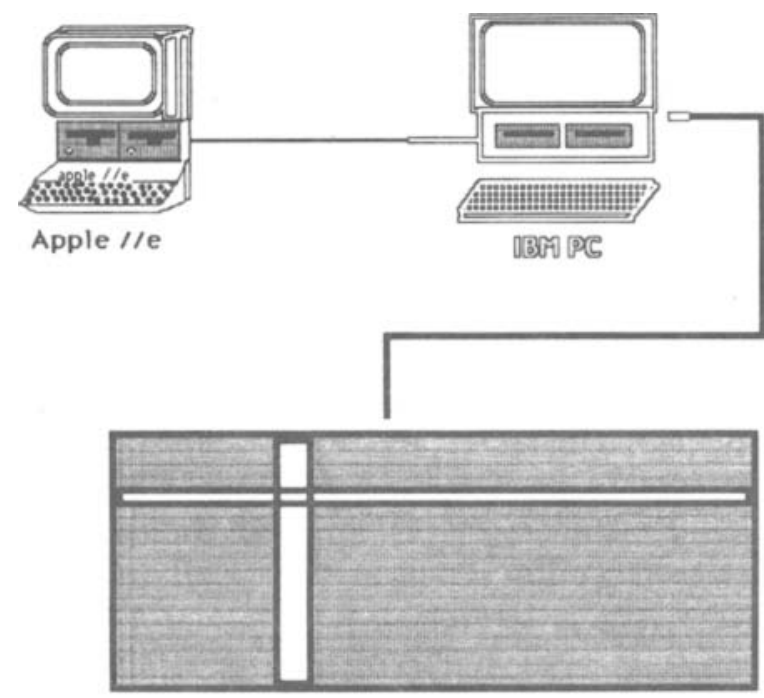

IBM 3031 MAINFRAME

Figure 2. Simple telecommunications network by 300-baud modem. 


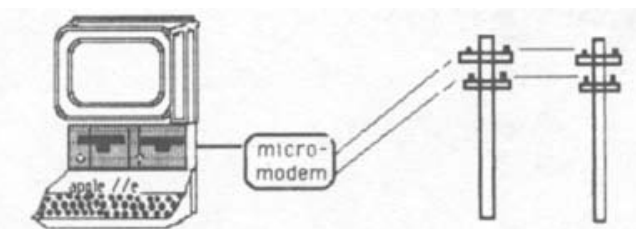

Apple //e
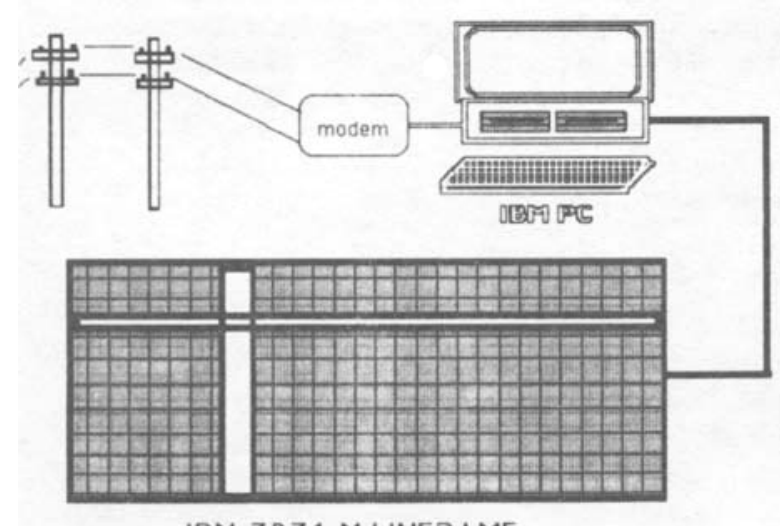

IBM 3031 MAINFRAME

Figure 3. Direct 9600-baud transfer network.

ditory modality. The Apple computer serves as a frontend processor or stand-alone computer. Data that are collected are uploaded to the IBM mainframe for storage. Large-scale data transformation or retrieval can be easily performed, and the results can be downloaded to the Apple. The advantages of this are obvious. Off-line preprocessing by the Apple does not demand a lot of mainframe processor time. The Apple is not in competition with other users for processor time, and more users can operate on the system. This in turn allows faster response by the mainframe.

The efficacy of biofeedback as a useful clinical technique can now be investigated and replicated with a larger number of individuals. Programs for these computers ensure consistency in data collection and in treatment protocol presentation. However, the Apple //e is still somewhat limited in terms of memory and speed to handle large data arrays needed for statistical processing. Although many statistical programs are being developed for the Ap- ple, these programs are generally for data reduction or simple statistical manipulations. At the present, there is still a great need for mainframe capability and statistical packages such as SAS and SPSS.

As we have demonstrated, even a small clinic with a limited budget can network with a larger mainframe for large-scale data-processing capabilities. If one is sending small numbers of data, then many of the commercially available communications programs will allow file transfer via modems over telephone lines to other systems. One disadvantage occurs when the data sets are large. Most low-cost modems are capable of data-transmission rates of only 300 or 1200 baud. If one is in a remote location, the slower data transfer will result in larger long-distance telephone charges. The alternative is to directly transmit data from the Apple to an IBM PC to convert the data from Apple DOS to IBM DOS. The IBM PC can then upload the data to a mainframe. Brown and Perez (1984) demonstrated the efficacy of the interface in the synthesis of research and clinical practice of computer-assisted biofeedback.

\section{REFERENCES}

Bremner, F. J., Yost, M., \& McKenzie, R. (1982). Computermanaged inferential statistical analysis of EEG data. Behavior Research Methods \& Instrumentation, 14, 300-302.

Brown, G. A. (1982). The efficacy of single subject statistics in acute neurophysiological preparations. Unpublished master's thesis, Trinity University, San Antonio, TX.

Brown, G. A., \& Perez, F. I. (1985). Single-subject statistical analysis of computer-assisted biofeedback. Behavior Research Methods, Instruments, \& Computers, 17, 327-330.

Carroll, D., \& Phys-Davies, L. (1979). Heartrate changes with exercise and voluntary heartrate acceleration. Biological Psychology, 8, 241-250.

Kewman, D. G., \& RoberTs, A. H. (1983). An altemative perspective on biofeedback efficacy studies. Biofeedback and Self Regulation, 8, 487-497.

LAVEREN, C., \& LUBIN, A. (1972). On planning psychophysiological experiments: Designs, measurement and analysis. In N. S. Greenfield \& R. A. Sternbach (Eds.), Handbook of psychophysiology. New York: Holt, Rinehart \& Winston.

Miller, N. E. (1982). Some directions for clinical and experimental research of biofeedback. In L. White \& B. Tursky (Eds.), Clinical biofeedback: Efficacy and mechanisms. New York: Guilford Press.

Steiner, S. S., \& DiNCE, W. M. (1981). Biofeedback efficacy studies: A critique of critiques. Biofeedback and Self Regulation, 6, 275-288. STEINER, S. S., \& DiNCE, W. M. (1983). A reply on the nature of biofeedback efficacy studies. Biofeedback and Self Regulation, 8, 499-503. 